\title{
Recurrence pattern and Survival analysis in patients undergoing radiotherapy IMRT technique for medulloblastoma
}

Hao Chang

Xi'an Tangdu Hospital of No4 Military Medical University

QiMing Wang

Xi'an Tangdu Hospital of No4 Military Medical University

ZongYan Yu

Xi'an Tangdu Hospital of No4 Military Medical University

ZiShen Zhang

Yan'an University

DeQuan Yu

Xi'an Tangdu Hospital of No4 Military Medical University

PinTing Zhao

Xi'an Tangdu Hospital of No4 Military Medical University

QiuJu Shao

Xi'an Tangdu Hospital of No4 Military Medical University

DongJie He ( $\sim$ hedongjie20031129@163.com )

Xi'an Tangdu Hospital of No4 Military Medical University

\section{Research}

Keywords: Medulloblastoma; Radiotherapy; Recurrence pattern; Survival analysis

Posted Date: May 19th, 2020

DOI: https://doi.org/10.21203/rs.3.rs-28813/v1

License: (1) (1) This work is licensed under a Creative Commons Attribution 4.0 International License.

Read Full License 


\section{Abstract}

Objective The technique of radiotherapy is associated with the recurrence pattern and prognosis of postoperative treatment for medulloblastoma. Our aim was to assess recurrence patterns and survival in patients with medulloblastoma between three-dimensional conformation (3D-CRT) and intensity modulated radiotherapy (IMRT). To analyze the factors affecting the prognosis, such as the use of 3DCRT or IMRT, the time interval of surgical and radiotherapy, whether there is chemotherapy and chemotherapy regimen.

Methods In this study, 36 patients with medulloblastoma were conducted investigating and data were analyzed from May 2009 to September 2017. The median survival of each group was calculated for different clinical stages,pathological types and treatment methods. The PFS and OS for patients curves were derived by Kaplan-Meier estimation, and which were calculated using the Cox proportional hazards model between the IMRT and 3D-CRT treatment groups.

Results Up to the follow-up date, 7 of the 9 patients in the 3D-CRT group had recurrence and 9 of the 27 patients in the IMRT group had recurrence. The 2-year and 5-year PFS of the IMRT group were $74.1 \%$ and $65.8 \%$, and which of the 3D-CRT group were $55.6 \%$ and $22.2 \%$, respectively. The PFS was significantly higher for patients treated with IMRT compared with 3D-CRT $(P<0.05)$. The 2-year overall survival rates of the IMRT group compared with the 3D-CRT group were the same, the 5-year overall survival rate of the IMRT group was higher than that of the 3D-CRT group, however, there was no statistical difference. The PFS and OS for patients with low-risk groups were better than those of the high-risk group $(P<0.05)$. There was no statistical difference between the M2 group and the M3 group in the 2-year and 5-year $\mathrm{OS}(\mathrm{P}>0.05)$. There was no statistical difference in the chemotherapy-free group, the EP chemotherapy regimen and the temozolomide (TMZ) chemotherapy group in the 2-year and 5-year PFS and OS $(P>0.05)$. The results of multivariate Cox proportional hazards model analysis showed that the residual, recurrence or metastasis before radiotherapy were independent prognostic factors affecting $P F S(P<0.05)$. Radiotherapy mode was negatively correlated, although there was no statistical significance $(P=0.061)$.

Conclusions IMRT can reduce the risk of spinal cord recurrence compared with 3D-CRT, especially the low rate of spinal cord recurrence. The development of the technique of radiotherapy was associated with improvements in medulloblastoma prognosis.

\section{Introduction}

Medulloblastoma (MB) is a central nervous malignancy that occurs in children and young adults. MB is frequently located in the posterior fossa. Radiotherapy of the craniospinal irradiation (CSI) techniques and chemotherapy are the standard treatments after operation of medulloblastoma [1].

To our knowledge,IMRT, volumetric-modulated arc therapy (VMAT), Tomotherapy (TOMO) and other modern radiotherapy technologies are obviously better than 3D-CRT and conventional radiotherapy technologies in terms of dose distribution in the target volume and normal tissue protection [2- 
4].Previous study have shown that 3D-CRT avoiding failures related to radiotherapy uncertainties to some extent, but there are still 1/3 failures statement[5]. Previous study had shown the pattern of failure associated with medulloblastoma patients treated with proton radiation therapy was similar to the pattern of failure in patients treated with photon radiation therapy [6].Howerver, there are few reports about recurrence patterns after radiotherapy.

In this paper, the recurrence patterns of 36 cases of medulloblastoma admitted to our hospital after radiotherapy were analyzed,and the survival was further analyzed to provide a clinical basis for the selection of radiotherapy techniques.

\section{Materials And Methods}

\section{Patients and treatments}

A total of 36 patients with medulloblastoma in hospital were enrolled and who were confirmed by pathology after surgery between May 2009 and September 2017.All patients received imaging of the entire neuraxis with magnetic resonance imaging (MRI) before craniospinal irradiation (CSI). Definitions of clinical target volume (CTV) are based on imaging aspects. We designed a clinical target volume (CTV) for CSI containing the spinal canal, which identified on computed tomography (CT) and T2-weighted magnetic resonance imaging (T2-MRI).Clinical data obtained from a retrospective review of charts including the patient's sex, age, risk status, interval time between surgery and radiation.After completion of radiation therapy, patients routinely underwent imaging of the entire neuraxis.Patients with evidence of recurrent disease were discussed in a multidisciplinary collaboration before salvage therapy.

\section{Radiation therapy and chemotherapy}

Patients were scanned using a GE Light Speed 16-slice computed tomography ( CT ) Scanner with 3.0$\mathrm{mm}$ slice thickness and were treated in the prone or supine position. The planned system used the Eclipse11.0 planned system (Varian Medical Systems Inc. Palo Alto, CA). A total of 2-3 isocenters were set.Patients with 3D-CRT plan were positioned in the prone position and the whole brain field was irradiated by two parallel pairs of traditional $90^{\circ}$ and $270^{\circ}$. The lower boundary of the whole brain field intersects with the upper boundary of the spinal field, and the whole brain field collimator rotates at a certain angle to match the scattered penumbra of the spinal field.The junction layer of the cerebral spinal cord underwent three $1 \mathrm{~cm}$ shifts (5th, 10th and 15th). Patients in IMRT group were supine or prone, and the spinal cord planning target volume (PTV) was designed with five field coplanar designs of $320^{\circ}, 340^{\circ}$, $0^{\circ}, 20^{\circ}$ and $40^{\circ}$, and the collimator angle was $0^{\circ}$. For patients with long PTV in the spinal cord, the PTV in the spinal cord was divided into upper and lower parts, and the overlap area of the upper and lower parts was about $2 \mathrm{~cm}$.In IMRT group, the angle of the two whole brain field collimator was about $85^{\circ}$ and $95^{\circ}$, which could optimize the dose of the cerebrospinal junction and reduce penumbra at the millimeter level, so that only one $1 \mathrm{~cm}$ shift was needed for the whole cerebrospinal junction. The dose distribution of 3DCRT plan and IMRT plan is shown in Fig 1. 
The radiotherapy dose was $26-36$ Gy in the whole brain and spinal cord, and the dosage of local tumor bed or residual and recurrent lesions was 54-60 Gy. The chemotherapy regimen was treated with 4-8 cycles of EP (Etoposide + Cisplatin) or 8-12 cycles of oral administration of temozolomide (TMZ) after craniospinal irradiation.

\section{Follow-up and measurements}

The imaging data of 36 patients with medulloblastoma were studied and evaluated by two experienced radiologists to observe the recurrence and metastasis after radiotherapy.The patients were followed up by telephone and examination at the hospital. PFS was defined as the time from the start of radiotherapy to the disease progression of the patient or the last follow-up.OS was defined as the time from the beginning of radiotherapy to the death of the patient or to the follow-up.The survival data in 3D-CRT group and IMRT group were analyzed.

\section{Statistical analysis}

All calculations were performed with SPSS version 22.0 for Windows software.The Kaplan-meier method was used for survival analysis, and the log-rank method was used to compare the survival differences between groups. The Cox proportional hazards regression model was used to determine prognostic factors on PFS and OS.Pख0.05 was considered statistically significant.

\section{Results}

\section{Patient characteristics}

Characteristics of the patients are shown in Tab 1.Among all 36 cases, 19 males(52.8\%) and 17 females $(47.2 \%)$, the age at diagnosis was from 3 years to 52 years old with a median age of 10.5 years old. The follow-up period was up to December 6,2019 , with a median follow-up period of 60.5 months (27.4-149.7 months). The median follow-up time of 3D-CRT group and IMRT group was 111.3 months (76.6-149.7 months) and 45.6 months (27.4-89.3 months),respectively.3-DCRT irradiation group was used from May 2009 to August 2013, and IMRT irradiation group was used from September 2013 to September 2017.The interval time of surgery and radiotherapy was 4 weeks, 8 weeks and 12 weeks. The examination before radiotherapy for tumor residue and spread was divided into non-spread group (M0), local residual group (M2) and cerebrospinal fluid spread (M3).EP regimen and TMZ regimen were used for adjuvant chemotherapy.

\section{Recurrence rules}

At the time of last follow-up, there were 7 cases of recurrence in the 3D-CRT group, including 4 cases of spinal cord spread,there were cases of spinal cord spread in the IMRT group and there was only 1 case of spinal cord spread. The disseminated magnetic resonance of the spinal cord presents as solitary single (Fig. 2a), or as diffuse meningeal thickening (Fig. 2c), or as space-occupying lesions in the spinal cord (Fig. 2b).There was 1 case of vertebral metastasis in the 3D-CRT group (Fig.2d), and the magnetic 
resonance T1 showed abnormally low signal shadow of multiple cones, while there were 2 cases of vertebral metastasis in the IMRT group, of which 1 case was shown in figure $2 \mathrm{e}$, and the PET/CT showed high metabolism of single vertebral body. There were 1 case of frontal lobe metastasis in the 3D-CRT group and 1 case in the IMRT group》respectively (Fig. $2 f$ and Fig. $2 g$ ). The remaining sites of recurrence were in situ (Fig. 2h) or intracranial disseminated lesions (Fig. 2i).

\section{Survival analysis}

According to the statistical analysis of the survival of 36 patients with medulloblastoma, the 2-year and 5-year PFS of the IMRT group were $74.1 \%$ and $65.8 \%$, respectively, while the 2-year and 5-year PFS of the 3D-CRT group were $55.6 \%$ and $22.2 \%$, respectively. The IMRT group was superior to the 3D-CRT group in PFS $(p<0.05)$.There was no difference between the 2 -year and 5 -year OS of the two groups $(p>$ 0.05). The results of the two groups are shown in Fig 3 and Tab 2.

Compared with interval ( 4 or 8 weeks) between surgery and radiotherapy,there was no statistical difference in both PFS and OS.The interval $<12 \mathrm{w}$ group was better than the $>12 \mathrm{w}$ group $(P<0.05)$, but there was no difference in OS between the two groups $(p>0.05)$. The 2-year and 5-year PFS of the nondisseminated group (M0) were better than the local residual group (M2) and the CSF disseminated group (M3) $(P<0.05)$. There was no statistical difference in PFS between M2 group and M3 group $(p>0.05)$. The M0 group was better than the M2 and M3 groups $(P<0.05)$,and there was no statistical difference in OS between the M2 and M3 groups $(p>0.05)$.There was no statistical difference among the chemotherapyfree group, EP chemotherapy regimen and TMZ chemotherapy group in the 2-year and 5-year PFS and OS groups $(p>0.05)$. The comparison of survival analysis of the above groups is shown in Tab 2.

Radiotherapy technique, interval time between surgery and radiotherapy,and whether residual, recurrence or metastasis before radiotherapy are the main factors affecting PFS. The results of multivariate Cox regression model analysis show that residual, recurrence or metastasis before radiotherapy is an independent prognostic factor affecting PFS $(P<0.05)$. However, radiotherapy mode was negatively correlated, although there was no statistical significance $(P=0.061)$.Cox regression analysis of each group is shown in Tab 3.

\section{Discussion}

Medulloblastoma (MB) is a common malignant tumor in children. It is a malignant and invasive embryonic tumor that occurs in the cerebellum or fourth ventricle and accounts for $12-25 \%$ of all CNS tumors. The main treatment of medulloblastoma is surgery. Postoperative radiotherapy can significantly reduce the recurrence and prolong the survival rate of patients. A recent study has shown that supine position for CSI was found to have similar survival outcomes compared with the prone position[7].A multicenter study found that modern radiotherapy techniques can enhance the dose uniformity in the target area, especially the spinal cord target area [2]. However,there is little research on survival related to technology. Our study found that the IMRT radiotherapy dose of spinal cord recurrence was only 1 case, while the 3DCRT group had a relatively high recurrence rate of 3 cases. A number of other studies have 
found that [8, 9], IMRT and VMAT overlap planning design allows for more positioning errors, which may be another advantage of IMRT in craniospinal irradiation. This is different from the pattern of recurrence after proton radiotherapy. One study found [6], the rate of extracranial recurrence, especially spinal cord recurrence, was higher after proton radiotherapy. However, our study suggested that the rate of spinal cord recurrence and metastasis after IMRT radiotherapy for medulloblastoma was very low. The previously reported incidence of vertebral metastasis is not high [10]. Our study suggests that vertebral metastasis is not uncommon, and various imaging techniques such as MR and PET-CT can play a role in diagnosis.

It was found that the following factors are important in rate of survival: radiotherapy technique, time interval between surgery and radiotherapy, assessment of pre-radiotherapy, chemotherapy intervention.

Firstly,whole brain and spinal cord technique is safe and feasible to use whole brain field irradiation. One study found [5], the application of left and right brain field in the whole brain and spinal cord was effective for penetrating irradiation, and only 1 of the 76 patients with 3D-CRT technology developed the failure of the sieve plate area. However, our study found that 2 out of 36 patients with medulloblastoma developed frontal lobe metastasis, which was considered as a possible failure of the sieve plate area. With the progress of radiotherapy technology, especially the development of VMAT and TOMO program for the treatment of whole brain and spinal cord technology $[11,12]$, the dose distribution in the target region was more uniform than that of 3DCRT and IMRT.

Secondly,whether the time interval between surgery and radiotherapy affects prognosis is still controversial.Del Charco[13] indicated that the 5 -year local control rate of $<45$ days was $89 \%$, while that of $>45$ days was only $68 \%$. And another study had shown that early postoperative radiotherapy could even be harmful[14]. The 5 -year PFS was $0 \%, 85 \%$, and $75 \%$, respectively, for intervals from surgery to radiotherapy of $<3$ weeks, $3-5$ weeks and $\geq 6$ weeks was $0 \%, 85 \%$, and $75 \%$, respectively. Our study found that the PFS was significantly shortened when the interval time between surgery and radiotherapy was more than 12 weeks, suggesting that the interval time between surgery and radiotherapy for medulloblastoma should not be more than 12 weeks at the latest.

Thirdly,radiotherapy is an important part of the comprehensive treatment of medulloblastoma. The presence or absence of postoperative residual and spread is an important factor affecting the prognosis [15]. The prognosis of patients is poor when it is disseminated and planted along the cerebrospinal fluid. According to the different states of Chang's staging system before radiotherapy [16],the patients were divided into stage M0 - stage M4. M0: no tumor metastasis was found;M1: only tumor cells were found in cerebrospinal fluid;M2: tumor cells are confined to the brain;M3: presence of CSF metastasis;M4: there are distant metastases outside the nervous system. The survival conditions were markedly different from period to period. Our study also found that the survival of the non-disseminated group (M0) was the best, while the survival of the partial residual group (M2) and the CSF disseminated group (M3) was poor. Since all the patients in this study were aged $\geq 3$ years, the M0 group was the low-risk group, while the M2 groups and the M3 groups were the high-risk groups. Modern installments have been added to 
molecular biology's classification in a more refined way [17], and molecular typing was not studied in this study due to the defects of the samples.

Lastly,Postoperative chemotherapy is an important part of the comprehensive treatment of medulloblastoma, and a number of studies have suggested that postoperative chemotherapy can improve the survival of patients with medulloblastoma $[18,19]$. Research from National Cancer Data Base shown that[19], the 5-year survival rate of the adjuvant chemotherapy group was significantly higher than that of radiotherapy alone, which was $86.1 \%$ and $71.6 \%$, respectively. Recent research suggests that preoperative chemotherapy can also yield survival benefits [20].In terms of the choice of chemotherapy regimen, the combination of three drugs may be more effective [21],lomustine(CCNU), cisplatin, and vincristine or cyclophosphamide, cisplatin, and vincristine are more commonly used.Our study indicated that there was no statistical difference in PFS and OS in the chemotherapy group,the chemotherapy-free group,the EP chemotherapy group and the TMZ chemotherapy group. Our study also clarified that single drug or double drug combination chemotherapy could not make patients with medulloblastoma benefit from postoperative chemotherapy.

Multivariate Cox regression analysis showed that recurrence, residual or spread before radiotherapy was an independent prognostic factor affecting PFS.This also explained, to some extent, the significant shortening of PFS in patients with medulloblastoma more than 12 weeks after surgery and radiotherapy, which may be due to the increased proportion of local recurrence or spinal cord dissemination due to the extended interval, thus affecting the disease-free survival of patients. However, radiotherapy is a negative correlation factor, indicating that IMRT technology can reduce the negative survival effect caused by recurrence spread to a certain extent.

In summary, IMRT technology has advantages over 3D-CRT technology in total cerebral spinal radiation, especially the lower spinal cord recurrence rate. The interval time between surgery and radiotherapy should be no more than 12 weeks.PFS and OS were higher in patients without residual, recurrence and spread before radiotherapy. Chemotherapy with TMZ alone or EP combined with both drugs did not increase survival. Multivariate analysis showed that the pre-radiotherapy status was an independent factor, and IMRT technology could reduce this survival effect. This study is a retrospective analysis. There

may be a certain selection bias, no molecular typing analysis. We expect a large scale of domestic case registration and follow-up observation to obtain more comprehensive and reliable clinical data, so as to provide a more reliable basis for our clinical decision.

\section{Abbreviations}

IMRT:intensity modulated radiotherapy; 3D-CRT:three-dimensional conformation ; VMAT:volumetricmodulated arc therapy; TOMO:tomotherapy ;PFS: progression-free survival; OS: overall survival; MB:medulloblastoma ; TMZ :temozolomide; MRI :magnetic resonance imaging; CT:computed tomography; CSI:craniospinal irradiation; CTV:clinical target volume; PTV:planning target volume; 


\section{Declarations}

\section{Ethics approval and consent to participate}

Not applicable.

\section{Consent for publication}

Consent for scientific usage of clinical data was obtained from all patients included in the study.

\section{Availability of supporting data}

Not applicable.

\section{Competing interests}

The authors declare that they have no competing interests.

\section{Funding}

Not applicable.

\section{Authors' contributions}

Hao Chang and DongJie He drafted the manuscript. ZiShen Zhang improved the patients immobilization. QiMing Wang, ZongYan Yu, participated in data collection and helped to analyze the data. DeQuan Yu, PinTing Zhao prepared the plan design. QiuJu Shao participated in the coordination of the study. All authors read and approved the final manuscript.

\section{Acknowledgements}

We thank Feng Xiao from Department of Radiation Oncology, XiJing Hospital of Air Force Medical university, for the plan quality assurance support.

\section{References}

1. Millard NE, De Braganca KC. Medulloblastoma. J Child Neurol. 2016;31:1341-53.

2. Seravalli E, Bosman M, Lassen-Ramshad Y, Vestergaard A, Oldenburger F, Visser J, et al. Dosimetric comparison of five different techniques for craniospinal irradiation across 15 European centers: analysis on behalf of the SIOP-E-BTG (radiotherapy working group). Acta Oncol. 2018;57:1240-9.

3. Wong KK, Ragab O, Tran HN, Pham A, All S, Waxer J, et al. Acute toxicity of craniospinal irradiation with volumetric-modulated arc therapy in children with solid tumors. Pediatr Blood Cancer. 2018;65:e27050. 
4. Myers PA, Mavroidis P, Papanikolaou N, Stathakis S. Comparing conformal, arc radiotherapy and helical tomotherapy in craniospinal irradiation planning. J Appl Clin Med Phys. 2014;15:4724.

5. Skowronska-Gardas A, Chojnacka M, Morawska-Kaczynska M, Perek D, Perek-Polnik M. Patterns of failure in children with medulloblastoma treated with 3D conformal radiotherapy. Radiother Oncol. 2007;84:26-33.

6. Sethi RV, Giantsoudi D, Raiford M, Malhi I, Niemierko A, Rapalino O, et al. Patterns of failure after proton therapy in medulloblastoma; linear energy transfer distributions and relative biological effectiveness associations for relapses. Int J Radiat Oncol Biol Phys. 2014;88:655-63.

7. Verma J, Mazloom A, Teh BS, South M, Butler EB, Paulino AC. Comparison of supine and prone craniospinal irradiation in children with medulloblastoma. Pract Radiat Oncol. 2015;5:93-8.

8. Cao F, Ramaseshan R, Corns R, Harrop S, Nuraney N, Steiner P, et al. A three-isocenter jagged-junction IMRT approach for craniospinal irradiation without beam edge matching for field junctions. Int $\mathrm{J}$ Radiat Oncol Biol Phys. 2012;84:648-54.

9. Myers P, Stathakis S, Mavroidis P, Esquivel C, Papanikolaou N. Evaluation of localization errors for craniospinal axis irradiation delivery using volume modulated arc therapy and proposal of a technique to minimize such errors. Radiother Oncol. 2013;108:107-13.

10. Nikitovic M, Bokun J, Paripovic L, Golubicic I, Grujicic D, Sopta J. Bone metastases in medulloblastoma-single institution experience. Pediatr Hematol Oncol. 2013;30:80-91.

11. Zong-Wen S, Shuang-Yan Y, Feng-Lei D, Xiao-Long C, Qinglin L, Meng-Yuan C, et al. Radiotherapy for Adult Medulloblastoma: Evaluation of Helical Tomotherapy, Volumetric Intensity Modulated Arc Therapy, and Three-Dimensional Conformal Radiotherapy and the Results of Helical Tomotherapy Therapy. Biomed Res Int. 2018;2018:9153496.

12. Sarkar B, Munshi A, Ganesh T, Manikandan A, Mohanti BK. Dosimetric comparison of short and full arc in spinal PTV in volumetric-modulated arc therapy-based craniospinal irradiation. Med Dosim. 2019.

13. del Charco JO, Bolek TW, McCollough WM, Maria BL, Kedar A, Braylan RC, et al. Medulloblastoma: time-dose relationship based on a 30-year review. Int J Radiat Oncol Biol Phys. 1998;42:147-54.

14. Abacioglu U, Uzel O, Sengoz M, Turkan S, Ober A. Medulloblastoma in adults: treatment results and prognostic factors. Int J Radiat Oncol Biol Phys. 2002;54:855-60.

15. Bouffet E. Management of high-risk medulloblastoma. Neurochirurgie. 2019.

16. Chang $\mathrm{CH}$, Housepian EM, Herbert C. Jr. An operative staging system and a megavoltage radiotherapeutic technic for cerebellar medulloblastomas. Radiology. 1969;93:1351-9.

17. Juraschka K, Taylor MD. Medulloblastoma in the age of molecular subgroups: a review. J Neurosurg Pediatr. 2019;24:353-63.

18. Atalar B, Ozsahin M, Call J, Napieralska A, Kamer S, Villa S, et al. Treatment outcome and prognostic factors for adult patients with medulloblastoma: The Rare Cancer Network (RCN) experience. Radiother Oncol. 2018. 
19. Kann BH, Lester-Coll NH, Park HS, Yeboa DN, Kelly JR, Baehring JM, et al. Adjuvant chemotherapy and overall survival in adult medulloblastoma. Neuro Oncol. 2017;19:259-69.

20. Moots PL, O'Neill A, Londer H, Mehta M, Blumenthal DT, Barger GR, et al. Preradiation Chemotherapy for Adult High-risk Medulloblastoma: A Trial of the ECOG-ACRIN Cancer Research Group (E4397). Am J Clin Oncol. 2018;41:588-94.

21. Packer RJ, Gajjar A, Vezina G, Rorke-Adams L, Burger PC, Robertson PL, et al. Phase III study of craniospinal radiation therapy followed by adjuvant chemotherapy for newly diagnosed average-risk medulloblastoma. J Clin Oncol. 2006;24:4202-8.

\section{Tables}

Tab 1 The clinical characteristics of the 36 patients with medulloblastoma

\begin{tabular}{|c|c|c|c|}
\hline Characteristics & Groups & $\mathrm{n}$ & Proportion $\square \%$ \\
\hline \multirow[t]{2}{*}{ Gender } & Male & 19 & 52.8 \\
\hline & Female & 17 & 47.2 \\
\hline \multirow[t]{2}{*}{ Radiation technical } & 3D-CRT & 9 & 25.0 \\
\hline & IMRT & 27 & 75.0 \\
\hline \multirow{2}{*}{ Time $^{a}$} & $\square 4 \mathrm{w}$ & 7 & 19.4 \\
\hline & $\square 4 \mathrm{w}$ & 29 & 80.6 \\
\hline \multirow[t]{2}{*}{ Time $^{b}$} & $\square 8 \mathrm{w}$ & 21 & 58.3 \\
\hline & $\square 8 \mathrm{w}$ & 15 & 41.7 \\
\hline \multirow[t]{2}{*}{ Time $^{\mathrm{C}}$} & $\square 12 \mathrm{w}$ & 26 & 72.2 \\
\hline & $\square 12 \mathrm{w}$ & 10 & 27.8 \\
\hline \multirow[t]{3}{*}{ Status before radiation } & M0 & 18 & 50.0 \\
\hline & M2 & 12 & 33.3 \\
\hline & M3 & 6 & 16.7 \\
\hline \multirow[t]{3}{*}{ Chemotherapy } & None & 14 & 38.9 \\
\hline & $\mathrm{EP}$ & 16 & 44.4 \\
\hline & TMZ & 6 & 16.7 \\
\hline
\end{tabular}

${ }^{a}$.The interval time of surgery and radiotherapy is four weeks. ${ }^{b}$.The interval time of surgery and radiotherapy is eight weeks. ${ }^{c}$.The interval time of surgery and radiotherapy is twelve weeks.

Tab 2 Survival analysis of the 36 patients with medulloblastoma 


\begin{tabular}{|c|c|c|c|c|c|}
\hline Characteristics & groups & PFS (2y/5y,\%) & $P$ value & $\mathrm{OS}(2 \mathrm{y} / 5 \mathrm{y}, \%)$ & $P$ value \\
\hline \multirow[t]{2}{*}{ Radiation technical } & 3DCRT & $55.6 / 22.2$ & 0.043 & $88.9 / 44.4$ & 0.476 \\
\hline & IMRT & $74.1 / 65.8$ & & $88.9 / 62.1$ & \\
\hline \multirow[t]{2}{*}{ Time $^{\mathrm{a}}$} & $\square 4 \mathrm{w}$ & $100.0 / 80.0$ & 0.128 & $100.0 / 75.0$ & 0.148 \\
\hline & $\square 4 \mathrm{w}$ & $62.1 / 43.4$ & & $86.2 / 51.2$ & \\
\hline \multirow[t]{2}{*}{ Time $^{\mathrm{b}}$} & $\square 8 w$ & $81.0 / 43.8$ & 0.328 & $90.5 / 56.7$ & 0.576 \\
\hline & $\square 8 \mathrm{w}$ & $53.3 / 46.7$ & & $86.7 / 51.9$ & \\
\hline \multirow[t]{2}{*}{ Time $^{\mathrm{C}}$} & $\square 12 \mathrm{w}$ & $80.8 / 55.1$ & 0.027 & $88.5 / 62.5$ & 0.113 \\
\hline & $\square 12 \mathrm{w}$ & $40.0 / 30.0$ & & $90.0 / 40.0$ & \\
\hline \multirow[t]{3}{*}{ Status before radiation } & M0 & $88.9 / 82.5$ & $\begin{array}{l}0.003^{1} \\
0.004^{2} \\
\end{array}$ & $100.0 / 79.9$ & $\begin{array}{l}0.016^{3} \\
0.016^{4} \\
\end{array}$ \\
\hline & M2 & $58.3 / 22.2$ & 0.852 & $91.7 / 33.3$ & 0.727 \\
\hline & M3 & $33.3 / 33.3$ & & $50.0 / 50.0$ & \\
\hline \multirow[t]{3}{*}{ Chmotherapy } & None & $71.4 / 45.7$ & $\begin{array}{l}0.810^{5} \\
0.579^{6} \\
\end{array}$ & $85.7 / 40.4$ & $\begin{array}{l}0.809^{7} \\
0.664^{8} \\
\end{array}$ \\
\hline & $\mathrm{EP}$ & $68.8 / 48.9$ & 0.752 & $100 / 63.6$ & 0.808 \\
\hline & TMZ & $66.7 / 66.7$ & & $66.7 / 66.7$ & \\
\hline
\end{tabular}

${ }^{a}$.The interval time of surgery and radiotherapy is four weeks. ${ }^{\mathrm{b}}$.The interval time of surgery and radiotherapy is eight weeks. ${ }^{\mathrm{c}}$.The interval time of surgery and radiotherapy is twelve weeks.

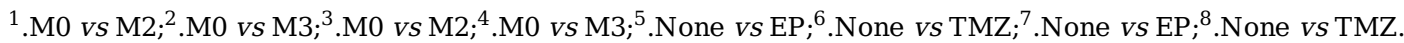

Tab 3 Multivariate Cox regression analysis of the PFS for patients with medulloblastoma

\begin{tabular}{lcccccc}
\hline & $\mathrm{B}$ & $\mathrm{SE}$ & Wald & $p$ & HR & $95 \%$ CI \\
\hline Interval time of surgery and radiotherapy & -0.962 & 0.513 & 3.514 & 0.061 & 0.382 & $0.140-1.045$ \\
\hline Status before radiation & 0.823 & 0.540 & 1.461 & 0.227 & 1.921 & $0.666-5.540$ \\
\hline
\end{tabular}

\section{Figures}



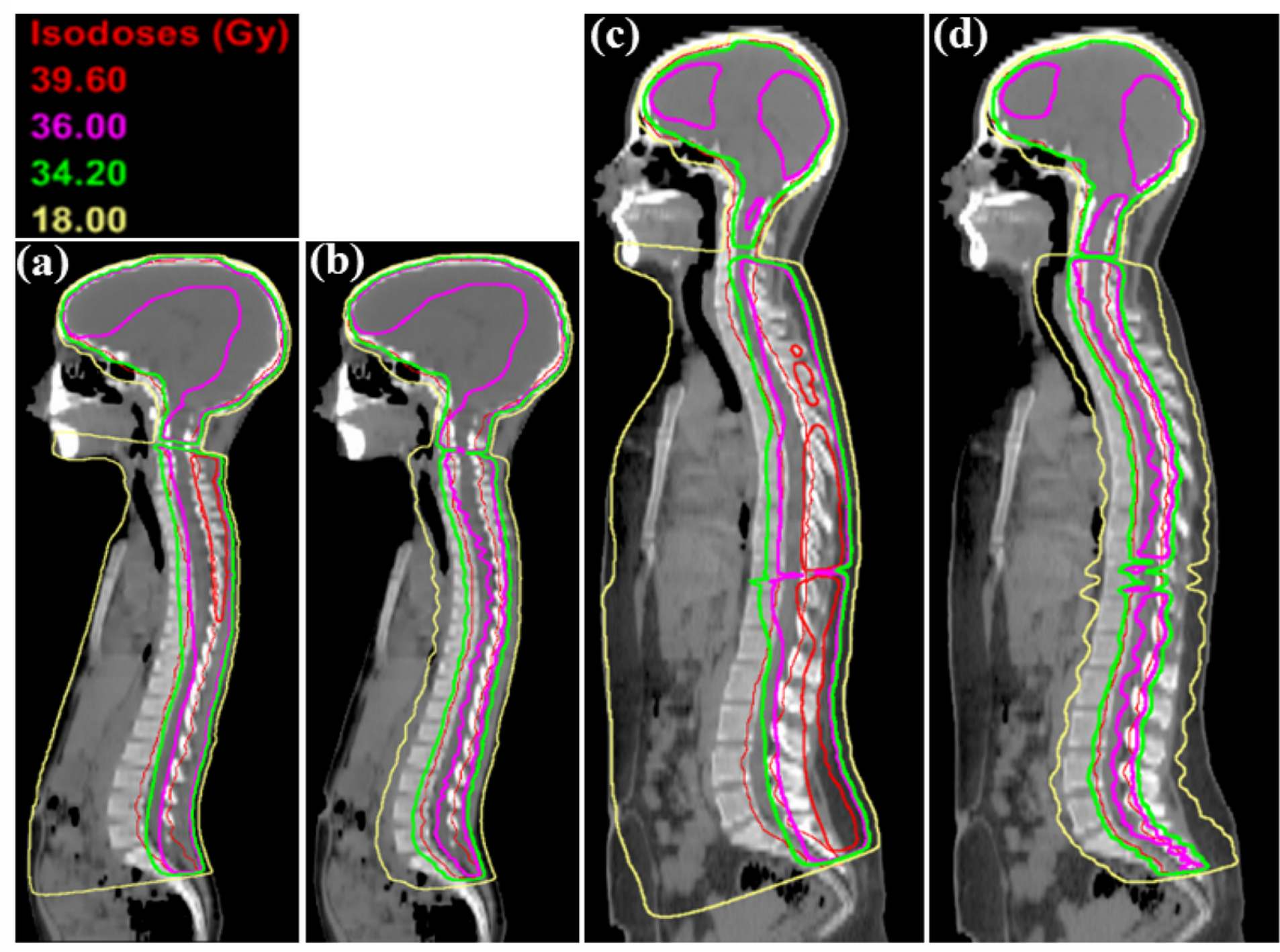

\section{Figure 1}

Sagittal view shows the dose distribution for two patients.(a) and (c) show doses distribution of 3-DCRT plan in children and adults. (b) and (d) show doses distribution of IMRT plans in children and adults. The yellow line represents the $18 \mathrm{~Gy}$ isodose curve,the green line represents the $34.2 \mathrm{~Gy}$ isodose curve,and the purple line represents the $34.2 \mathrm{~Gy}$ isodose curve. 

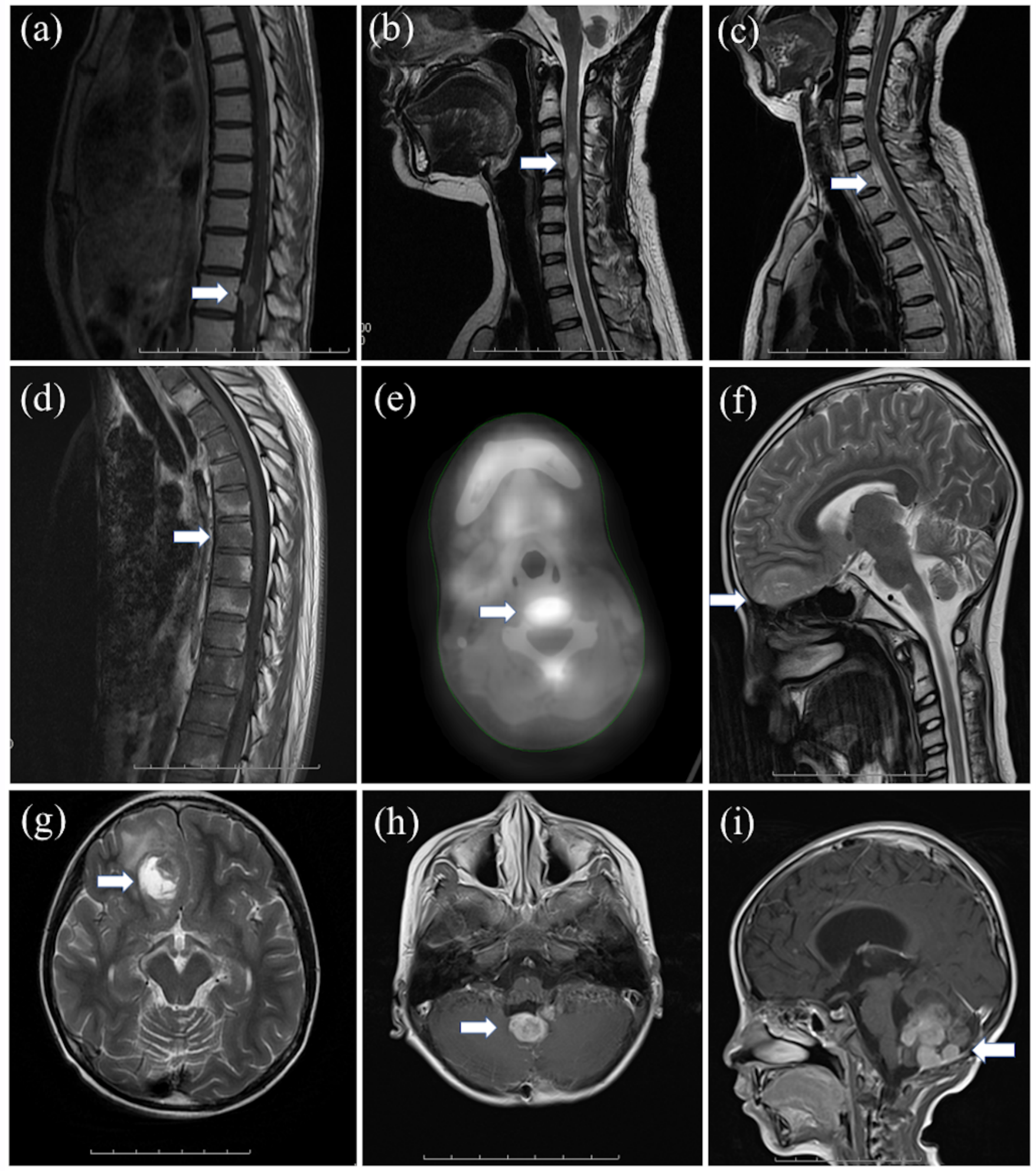

\section{Figure 2}

Relapse cases of medulloblastoma patients. $(a, b, c)$ MRI imaging data indicate cases of spinal cord spread.(d,e) Imaging data are shown for bone metastasis. $(f, g)$ MRI imaging data are shown patients with plate area recurred.(h,l) MRI imaging data are demonstrated Recurrent in situ and posterior fossa. The white arrow points to the site of tumor recurrence or metastasis. 

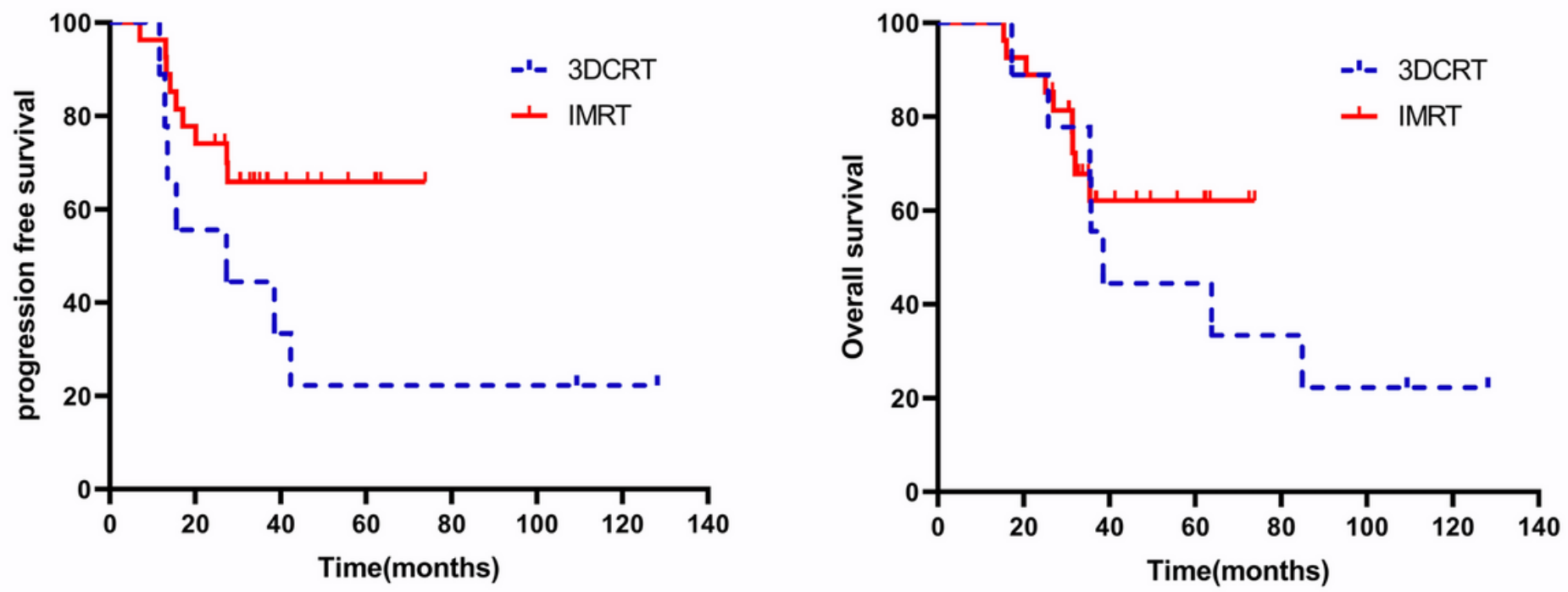

Figure 3

Survival outcomes. Survival curves showing progression-free survival and overall survival rates after radiation beginng. 